\title{
Direct oral anticoagulants in cancer-associated venous thromboembolism: It is high time for a change of therapeutic paradigm
}

\author{
Justyna Domienik-Karłowicz ${ }^{1}$, Miłosz Jaguszewski ${ }^{2}$, Marcin Kurzyna ${ }^{3}$ \\ ${ }^{1}$ Department of Internal Medicine and Cardiology, Medical University of Warsaw, Poland \\ ${ }^{2}$ First Department of Cardiology, Medical University of Gdansk, Poland \\ ${ }^{3}$ Department of Pulmonary Circulation, Thromboembolic Diseases and Cardiology, \\ Center of Postgraduate Medical Education, European Health Center, Otwock, Poland
}

This paper was guest edited by Prof. Krzysztof J. Filipiak

\begin{abstract}
Giancarlo Agnelli and colleagues have recently revealed results of the CARAVAGGIO study at the American College of Cardiology and World Congress of Cardiology's virtual scientific sessions. The outcomes of this trial, simultaneously published in the The New England
\end{abstract} Journal of Medicine [1],

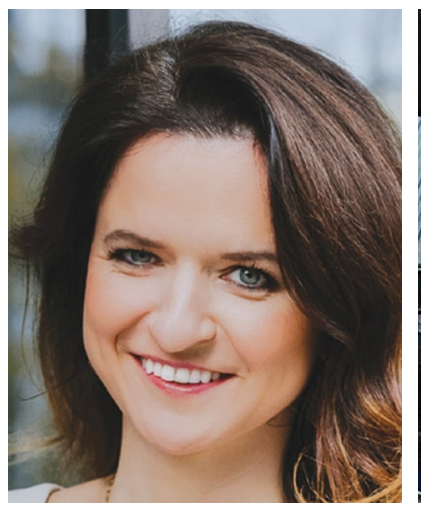
support the inclusion of apixaban as another direct oral anticoagulant (DOAC) for cancer patients with newly diagnosed incidental or symptomatic proximal deep vein thrombosis (DVT) or pulmonary embolism (PE). This study seems to finish the ongoing discussion on the possibility of using DOACs in cancer-associated thrombosis (CAT) and change the paradigm, setting the low-molecularweight heparin (LMWH) as a drug of choice and gold standard in this indication.

Venous thromboembolism (VTE) is the secondleading cause of mortality in cancer patients receiving chemotherapy [2]. The adjusted occurrence of VTE is a significant predictor of limited survival within the first year for all cancer types (HR 1.6-4.2; $\mathrm{p}<0.01$ ) [3]. Moreover, the risk of VTE varies depending on the type of cancer, the stage of metastatic disease, and receipt of certain anti-cancer therapies [4]. The highest risks apply to pancreatic cancer, he-
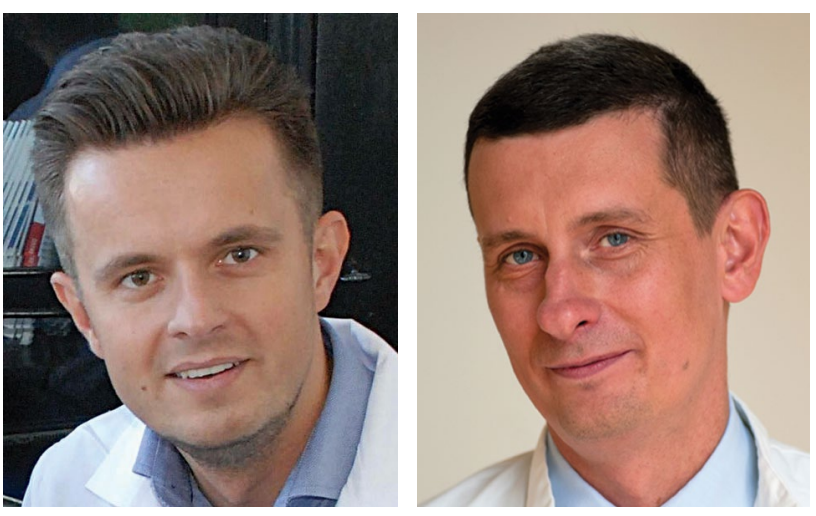

matological malignancy, lung, gastric, and brain cancer. The CLOT trial, published in 2003, showed superior efficacy of dalteparin compared to vitamin $\mathrm{K}$ antagonists in the prevention of recurrence of cancer-associated VTE and similar bleeding rates in both groups [5]. It was the basis for explicit recommendations for initial and long-term CAT treatment with LMWH in the following years. Recently the European Society of Cardiology Guidelines suggested that "edoxaban or rivaroxaban should be considered as an alternative to LMWH, with a word of caution for patients with gastrointestinal cancer due to the increased bleeding risk with DOACs" [6]. The latter was added to underline that the increased risk of bleeding may counterbalance the benefits of anticoagulation therapy in cancer patients. The risk of bleeding in patients treated with DOACs is an essential problem in "mucosal" types of cancer occurring in the gastrointestinal tract and urinary system.

Address for correspondence: Justyna Domienik-Karłowicz, MD, PhD, Department of Internal Medicine and Cardiology, Medical University of Warsaw, ul. Lindleya 5, 02-005 Warszawa, Poland, e-mail: jdomienik@tlen.pl 
Last, but not least, is the possibility of unfavorable drug interactions of DOACs with chemotherapy, which may occur during concomitant use.

In the Hokusai VTE Cancer study, oral edoxaban was confirmed to be noninferior to subcutaneous dalteparin concerning the composite outcome of recurrent VTE or major bleeding, but the rate of major bleeding was higher with edoxaban than with dalteparin [7]. Similarly, SELECT-D trial rivaroxaban has shown low VTE recurrence but the results for major bleeding were $4 \%$ for dalteparin and $6 \%$ for rivaroxaban. Unfortunately, the adequate rates for clinically relevant non-major bleeding were $4 \%$ for dalteparin and $13 \%$ for rivaroxaban [8]. Notably, both studies did not exclude patients with cerebral metastases nor brain tumors.

The newly published CARAVAGGIO study is a multinational, randomized, open-label, investigator-initiated study to compare efficacy and safety of apixaban and dalteparin in 1170 cancer patients with VTE [1]. Patients with basal-cell or squamous-cell carcinomas of the skin, primary brain tumors, brain metastases, and acute leukemias were excluded, but the study was not exclusive any other types of neoplastic disease. Approximately one-third of patients presented cancer at gastrointestinal sites. 585 patients were assigned to receive apixaban at a dose of $10 \mathrm{mg}$ twice daily for the first week and $5 \mathrm{mg}$ twice daily subsequently and 585 patients were assigned to receive dalteparin at a dose of $200 \mathrm{IU} / \mathrm{kg}$ of body weight subcutaneously once daily for the first month and $150 \mathrm{IU} / \mathrm{kg}$ of bodyweight subsequently over a period of 5 months.

The primary efficacy end-point was defined as the recurrence of VTE. The principal safety outcome was defined as major bleeding. Finally, 1155 patients were included in further analysis. Patients with $\mathrm{PE}$ accounted for $52.8 \%$ and $57.7 \%$ in the apixaban and dalteparin groups, respectively. Approximately $20 \%$ of the cases were individuals with incidental DVT or PE who were diagnosed during diagnostic procedures performed for reasons other than clinical suspicion of VTE. 97\% of cases presented active cancer at enrollment.

The primary efficacy end-point occurred in $5.6 \%$ patients in the apixaban group (32 patients: 13 recurrent DVTs, 19 recurrent $P E$, including 4 cases of fatal PE) compared to $7.9 \%$ (46 patients: 15 recurrent DVT, 32 recurrent $\mathrm{PE}$, including 3 cases of fatal $\mathrm{PE}$ ) in the dalteparin group $(\mathrm{p}<0.001$ for noninferiority; $\mathrm{p}=0.09$ for superiority in favor of apixaban).

Major bleeding occurred in 3.8\% (22) of patients in the apixaban group and $4 \%$ (23) of patients in the dalteparin group. Moreover, major gastroin- testinal bleeding occurred in $1.9 \%$ (11) of patients in the apixaban group and 1.7\% (10) patients in the dalteparin group. There were 2 fatal bleeding episodes in the dalteparin group compared to 0 in the apixaban group. Moreover, the rates of clinically relevant non-major bleeding were not significantly higher with apixaban group versus dalteparin (9\% and 6\%). The incidence of death was similar in both groups $-23.4 \%$ in the apixaban versus $26.4 \%$ in the dalteparin group. Most deaths were related to cancer $-85.2 \%$ and $88.2 \%$, respectively.

The key finding of this study was that oral apixaban is non-inferior to subcutaneous dalteparin for the treatment of VTE in cancer patients up to 6 months after diagnosis. Contradictorily to previous studies involving other DOACs, the occurrence of major bleedings, either general or gastrointestinal, were similar in apixaban and dalteparin groups. The clinical benefit of treatment prolonged for more than 6 months should be assessed in additional studies.

Cancer patients need to have access to a convenient and safe drug. The treatment regimen for VTE with apixaban and rivaroxaban allows for administering oral medications from the first day of therapy, which is exceptionally convenient in cases of DVT and low-risk incidental PE. However, it also requires a physician to make the right decision regarding the choice of DOAC or LMWH based on the type of cancer, comorbidities, bleeding risk, concomitant chemotherapy, and patient preference. The satisfactory safety profile of apixaban showed in the CARAVAGGIO trial, makes this choice easier. However, this work is not yet complete. More extensive research is still needed to allow this heterogeneous group of patients to be treated in a more individualized way, ensuring a satisfactory balance between antithrombotic efficacy and bleeding risk.

\section{Conflict of interest: None declared}

\section{References}

1. Agnelli G, Becattini C, Meyer G, et al. Caravaggio Investigators. Apixaban for the treatment of venous thromboembolism associated with cancer. N Engl J Med. 2020; 382(17): 1599-1607, doi: 10.1056/NEJMoa1915103, indexed in Pubmed: 32223112.

2. Kuderer NM, Ortel TL, Francis CW. Impact of venous thromboembolism and anticoagulation on cancer and cancer survival. J Clin Oncol. 2009; 27(29): 4902-4911, doi: 10.1200/ JCO.2009.22.4584, indexed in Pubmed: 19738120.

3. Chew HK, Wun T, Harvey D, et al. Incidence of venous thromboembolism and its effect on survival among patients with common cancers. Arch Intern Med. 2006; 166(4): 458-464, doi: 10.1001/ archinte.166.4.458, indexed in Pubmed: 16505267.

4. Horsted F, West J, Grainge MJ. Risk of venous thromboembolism in patients with cancer: a systematic review and meta- 
analysis. PLoS Med. 2012; 9(7): e1001275, doi: 10.1371/journal. pmed.1001275, indexed in Pubmed: 22859911.

5. Lee AYY, Levine MN, Baker RI, et al. Low-molecular-weight heparin versus a coumarin for the prevention of recurrent venous thromboembolism in patients with cancer. N Engl J Med. 2003; 349(2): 146-153, doi: 10.1056/NEJMoa025313, indexed in Pubmed: 12853587.

6. Konstantinides SV, Meyer G, Becattini C, et al. 2019 ESC Guidelines for the diagnosis and management of acute pulmonary embolism developed in collaboration with the European Respira- tory Society (ERS). Eur Heart J. 2020; 41(4): 543-603, indexed in Pubmed: 31504429.

7. Raskob GE, van Es N, Verhamme P, et al. Edoxaban for the treatment of cancer-associated venous thromboembolism. N Engl J Med. 2018; 378(7): 615-624, doi: 10.1056/NEJMoa1711948, indexed in Pubmed: 29231094.

8. Young AM, Marshall A, Thirlwall J, et al. Comparison of an oral factor xa inhibitor with low molecular weight heparin in patients with cancer with venous thromboembolism: results of a randomized trial (SELECT-D). J Clin Oncol. 2018; 36(20): 2017-2023, doi: 10.1200/JCO.2018.78.8034, indexed in Pubmed: 29746227. 